\title{
Power-law characteristics of damage and failure of ceramic coating systems under three-point bending
}

\author{
L.H. Liang *, X.N. Li, H.Y. Liu, Y.B. Wang, Y.G. Wei * \\ LNM, Institute of Mechanics, Chinese Academy of Sciences, Beijing 100190
}

\section{A R T I C L E I N F O}

\section{Article history:}

Received 25 September 2015

Revised 21 November 2015

Accepted in revised form 23 November 2015

Available online 28 November 2015

\begin{abstract}
A B S T R A C T
Damage and failure of ceramic coatings bonded on alloy substrates was studied by observing crack evolution in the coating systems under in situ three-point bending tests with corresponding load-displacement curves. A damage and catastrophic failure model on the ceramic coatings was proposed based on our experimental results and the Taylor expansion of the controlling variable. The results indicate that the damage increases with increasing stress and obeys the power-law characteristics with the power exponent of 0.5 , and the damage is 1 as the stress reaches the critical point corresponding to the failure of the coating systems. The damage rate increases rapidly when the stress is near the failure point and shows a power law singularity of -0.5 . The experimental results of thin, thick, nanostructured, and conventional micrometer-scale microstructured coatings are all in agreement with the predictions based on the model.
\end{abstract}

C 2015 Elsevier B.V. All rights reserved.

\section{Introduction}

Ceramic coatings are widely used in mechanical engineering, chemical engineering, aerospace and marine fields, etc. due to their high melting point and excellent resistance to erosion and wear. For example, ceramic coatings of several hundreds of micrometers are often sprayed or deposited on alloy substrates for thermal protection of blades of aerospace engines [1]. Once the ceramic coatings fracture and spall, the blades exposed high temperature will failure. Therefore, the study on fracture and damage of the ceramic coatings attracts great attention. Zhou et al. studied the fracture characteristics and the crack evolution in the ceramic top coatings for two-layer and multilayer systems under uniaxial tension and four-point bending [2]. They found that the transverse crack (vertical to the interface) density tended saturate as the strain reached a certain value [2]. Qian et al. found similar phenomena in studying the tensile damage behavior of a sandwiched coating system, and proposed a transverse crack evolution mode of the initiation, multiplication and saturation with increasing strain [3]. McGuigan et al. developed an elastic-plastic shear lag model describing cracking under uniaxial tensile strain of a brittle thin film on a deformable substrate and proposed that the (transverse) crack density is a function of applied strain [4]. Thouless et al. studied how the crack modes in films depend on material properties and thickness ratio by numerical analysis related to energy release rate [5-6]. Renusch and Schütze studied the damage kinetics of thermal barrier coatings by

\footnotetext{
* Corresponding authors.

E-mail addresses: lianglh@Inm.imech.ac.cn (L.H. Liang), ywei@lnm.imech.ac.cn (Y.G. Wei).
}

acoustic emission analysis of transverse and interface crack evolution, proposed a super-parabolic and accelerating linear dependence of the damage on the thermal cycling oxidation time, respectively, before and during spallation of the coatings, and demonstrated the power law characteristics of the damage with increasing temperature difference [7]. Trunova et al. studies the damage and failure characteristics of thermal barrier coatings by observing interface crack evolution with increasing high temperature oxidation time [8]. Brodin et al. studied fatigue damage of thermal barrier coatings by analyzing interface crack length change with increasing thermal cycling number [9].

It is clear, when ceramic coating/metallic substrate systems are subjected to bending, tension (mechanical load), high temperature oxidation, or thermal shock (thermal load), the microscopic transverse or interface cracks appear in the ceramic coating systems with increasing load or cycling number (for cycling load), i.e., the damage initiates, the number of the transverse cracks saturates and the interface fractures when the load reaches the maximum allowed value or the cycling number reaches the failure critical number [2-4,7-9]. As above indicated, researchers observed the change of the number of cracks or the crack length with strain, time, temperature or cycling number [2-4,7-9]. For thicker coatings or pre-oxidized systems, interface fracture between the coating and the substrate occurs, and the interface crack evolution was studied in particular for the coating systems under mechanical or thermal loading [7-11]. Recently, Zhou et al. studied in detail the damage and fracture of thermal barrier coatings by coupled acoustic emission and digital image correlation techniques [12], and found a similar damage behavior as in the Renusch and Schütze's study [7]. When the controlling variable (time or transverse crack density) reaches a critical value, the damage is 1 , after that the damage is even larger than 1 and 
increases sharply, which corresponds to the spallation or delamination of the coatings (i.e., interface fracture), before that the transverse crack evolution corresponds to the damage range from 0 to 1 [7,12]. Although the previous works describe effectively the damage characteristics of ceramic coatings to some degree, the damage larger than 1 is peculiar and a different model on the damage and fracture of the ceramic coatings reflecting physical mechanism is desired. Since ceramics are brittle, the fracture and failure maybe catastrophic, if a scaling law of damage and failure of the ceramic coatings can be developed, it will be significant not only in scientific understanding of coatings fracture but also in prediction of failure.

In this paper, the crack evolution of $\mathrm{ZrO}_{2}$ ceramic coatings with different thicknesses and microstructures, bonded on the same Ni-based superalloy substrates, under three-point bending were real time observed by in-situ scanning electron microscope. The damage evolution of the ceramic coatings, represented by the transverse or interface crack length, was studied with increasing tensile or shear stress for thin and thick coating systems, respectively, and a power law of the damage was developed based on the experimental results of all kinds of coatings and a unified theoretical analysis resulted from the Taylor's expansion. The scaling law of the damage rate of ceramic coatings, helpful for predicting the catastrophic fracture of coating systems, was revealed.

\section{Experimental}

\subsection{Specimen preparation}

The thermal barrier coating samples used in this study consist of YSZ ( $8 \mathrm{wt}$.\% $\mathrm{Y}_{2} \mathrm{O}_{3}$ stabilized $\mathrm{ZrO}_{2}$ ) top coatings prepared by the standard atmospheric plasma spraying method [13-14], NiCrAlY (25.42wt\%Cr$5.1 \mathrm{wt} \% \mathrm{Al}-0.48 \mathrm{wt} \% \mathrm{Y}$ ) bond coatings prepared by the high velocity oxygen fuel method, and Ni-based superalloy substrates [14]. The detailed preparation process and the microstructure of the coatings were described in Ref. [14]. We prepared thick coating samples with a coating thickness of 350-490 $\mu \mathrm{m}$, and thin coating ones with a coating thickness of $150-200 \mu \mathrm{m}$. The coating thickness actually includes the thickness of the ceramic top coating and the thickness of the bond coating of about tens of micrometer to a hundred micrometer. For both the thin and thick coating samples, two kinds of ceramic coatings were prepared by the use of different raw powders. One kind of coating with grains of about 40-100 nm diameter prepared from nanostructured YSZ powder [14], is represented by $\mathrm{Nx}$ with $\mathrm{x}$ being the sample number. The other kind is a conventional coating with micrometer scale splat grains of $200 \mu \mathrm{m}$ diameter and $2 \mu \mathrm{m}$ thickness prepared from conventional YSZ powder $[1,14]$ and is represented by Mx. A single number $\mathrm{x}$ denotes the thin coating samples, such as N0, M3..., and a double number $\mathrm{x}$ represents the thick coating samples, such as M00, N30, etc. The samples had a fabricated length, width and thickness of about $15 \mathrm{~mm}, 3 \mathrm{~mm}$ and $1.5 \mathrm{~mm}$, respectively.

\subsection{In-situ three-point bending test}

The samples were grinded and polished, then were placed in the sample room of the FEI Sirion 400 NC scanning electron microscope (SEM) and were sandwiched in the designed jig for in-situ observation of crack initiation and propagation on the side face of the samples under loading by using the mechanical testing apparatus - Gatan Microtest 2000, as shown in Fig. 1 in Ref. [11]. The three-point bending measurements were carried out by a slight movement of the jig controlled by the mechanical testing apparatus and the load-displacement curves were obtained. The load was applied on the alloy substrate face, and two fulcrums contact the ceramic coating face, as shown on Fig. 1. The span was $10 \mathrm{~mm}$. The loading rate was $0.1 \mathrm{~mm} / \mathrm{min}$. The evolution of cracks on the side face with increasing load was observed and recorded by use of the SEM. At some loading points corresponding to the crack initiation

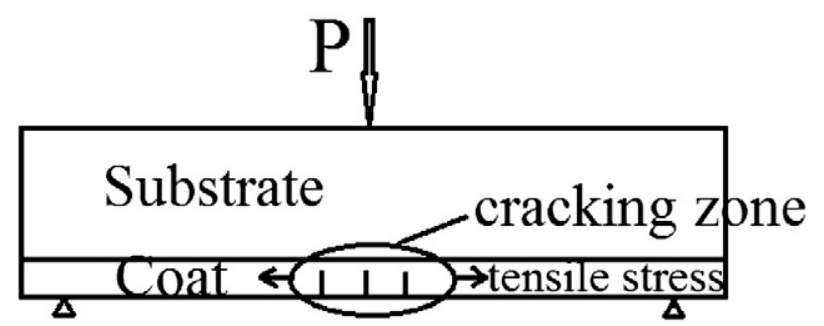

Fig. 1. Schematic of the coating/substrate systems under the three-point bending, the transverse cracks occur with loading for elastic brittle coatings.

and propagation, the loads were stopped temporarily to take pictures for obtaining the crack maps. After taking pictures at a displacement point, the load was resumed and applied continuously again. The crack length was calculated by image analyzing based on the side face micrographs of the coatings.

\section{Experimental results and damage model}

\subsection{Experimental results}

The load-displacement curve of each sample with a series of corresponding crack evolution maps can be obtained as shown in Ref. [11]; here two examples are shown in Figs. 2-3. Fig. 2(a) shows the load-displacement curve of the nanostructured thin coating sample No, from which it can be seen that the load increases with enhancing displacement up to the peak value. The process corresponds to the initiation, propagation and saturation of the transverse cracks in the coating, as shown in Fig. 2(b). After that the load drops with enhancing displacement, corresponding to the rupture of coating and cracks in the substrate. Note that the approximate plateau in the curve corresponds to plastic deformation of the substrate, and the cracks propagated through the interface and into the substrate, as shown in Fig. 2(c), which is not our focus. The crack maps before the peak load and close after the peak were paid attention. The elastic segment in the load-displacement curve was almost kept before the peak load. The tiny jumps in the curve correspond to the points of stopping the loading and taking pictures, during the process the load dropped slightly without change of displacement. The crack maps corresponding to some points in the load-displacement curve, such as the numbers 1-9 in Fig. 2(a) and the numbers 1-6 in Fig. 3(a), were real time obtained. For example, the crack maps corresponding to the numbers 2, 6, and 9 in Fig. 2(a) are shown in Fig. 2(b), which shows the initiation, multiplication and saturation of the cracks in the same position and is similar to the previous reports [3,11]. Note that Figs. 2(b), (c) and 3(b) only shows a part of all maps of the whole sample due to limited field of view. In fact, many pictures with different fields of views, corresponding to each point in the curve were taken.

It can be found from the experimental observation that the transverse crack, vertical to the interface between the coating and the substrate, initiates at some defect in the coating with loading, and multiple transverse cracks in the thin coatings emerge instantaneously and saturate soon when the load is close to a critical peak value corresponding to a tensile stress level of coating strength. For the thin coating systems, tensile failure dominates and the multiple transverse cracks emergence are the main fracture mode as analyzed in Ref. [11]; thus the length of the transverse cracks was measured when the damage was evaluated.

For the thick coating systems, interface shear failure dominates and interface fracture between the coatings and the substrates is the main fracture mode [11], as shown in Fig. 3(b), and thus the length of interface crack was calculated for describing damage. The load-displacement curves of the thick coatings show obvious load drop after the peak values compared to those of the thin coatings, as shown in Fig. 4 in 
a

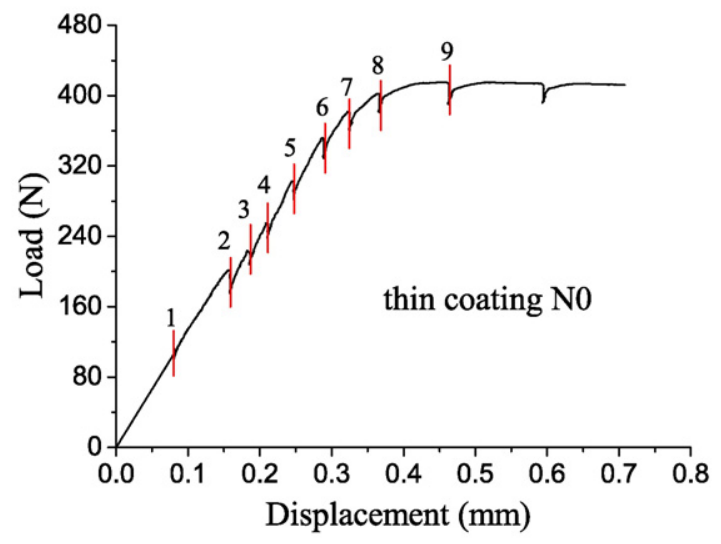

b
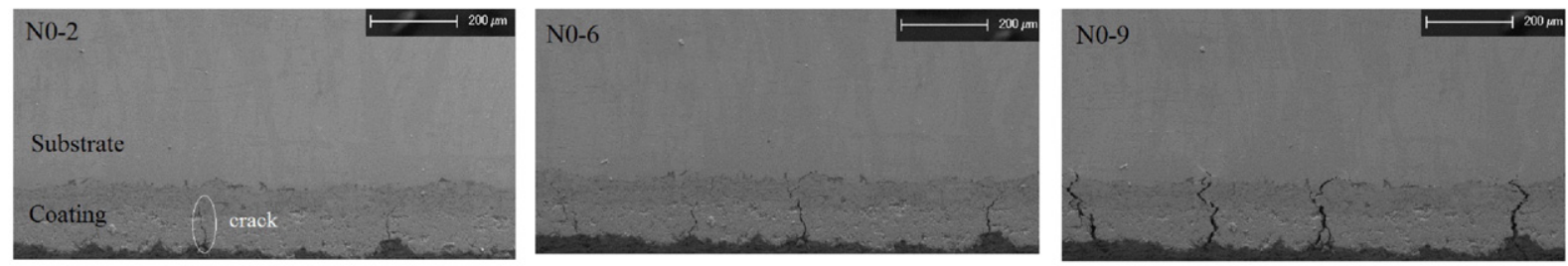

C

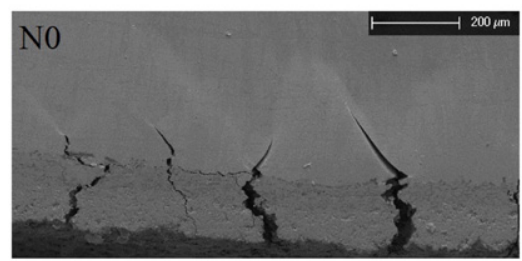

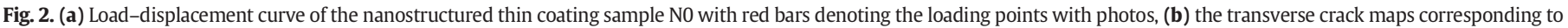
the points numbered by 2, 6, and 9 in the curve, a crack is circled to guide eyes, (c) the cracks propagate into the substrate after the peak stress.

Ref. [11], which implies the obvious catastrophic fracture characteristics of the thick coatings.

\subsection{Damage model}

(A) Damage definition: The initial crack length $L_{0}$ (at micrometer scale based on the SEM observation) in a coating of one sample under loading is defined as the initial damage, the total crack length $L_{f}$ in the coating at the failure load (i.e., the critical point of the load-displacement curve or the peak load) is defined as the complete damage. The initial damage variable $D_{0}=L_{0} / L_{f}$, and the complete damage variable $D_{f}=1$. For the thin coating systems, the multiple transverse cracks emergence in the coatings is the main fracture mode, and the tension failure dominates the coating systems, therefore, the tensile stress $\sigma$ in the coatings is taken as the controlling variable. The total transverse crack length $L$ in the thin coating corresponding to $\sigma$ is used to calculate damage variable $D=L / L_{f}$ with $L_{f}$ being the total transverse crack length corresponding to the failure stress $\sigma_{f}$ of the sample, and the initial tensile stress $\sigma_{0}$ corresponds to the initial transverse crack length $L_{0}$.

For the thick coating systems, the interface fracture between the coating and the substrate is the main fracture mode, and the shear failure dominates the coating systems, therefore, the shear stress $\tau$ at the coating interface is taken as the controlling variable, $\tau_{0}$ and $\tau_{f}$ are the initial and failure shear stress, respectively. The damage variable $D$ is calculated by use of the same method as above defined, but based on the interface crack length.

The coating/substrate systems are treated simply as a two-layer composite beam, as shown in Fig. 7 in Ref. [11], the calculation of $\sigma$ and $\tau$ of the coating at the coating interface can be obtained based on the composite beam model [11,15],

$\sigma=\frac{P l E_{c} \varsigma}{4\langle E I\rangle}$

$\tau=\frac{P E_{c}\left[\left(h_{c}+\varsigma\right)^{2}-\varsigma^{2}\right]}{4\langle E I\rangle}$,

where $P$ is the load, which is taken as the critical load $P_{f}$ at the critical point of the load-displacement curve, corresponding to the interface fracture for the thick coating systems or the penetrating (through the interface) of the transverse cracks for the thin coating systems, when the failure stress is calculated, $l$ is the span length, $\langle E I\rangle=E_{s} I_{s}+E_{c} I_{c}$ is the equivalent bending stiffness with the elastic modulus $E$ (the subscripts $c$ and $s$ represent the coatings and the substrates, respectively, $\left.E_{c}=100 \mathrm{GPa}, E_{s}=200 \mathrm{GPa}[11]\right), I_{S}=\int_{\varsigma-h_{S}}^{\varsigma} b y^{2} d y$ and $I_{c}=\int_{\varsigma}^{S+h_{c}} b y^{2} d y$ 
a

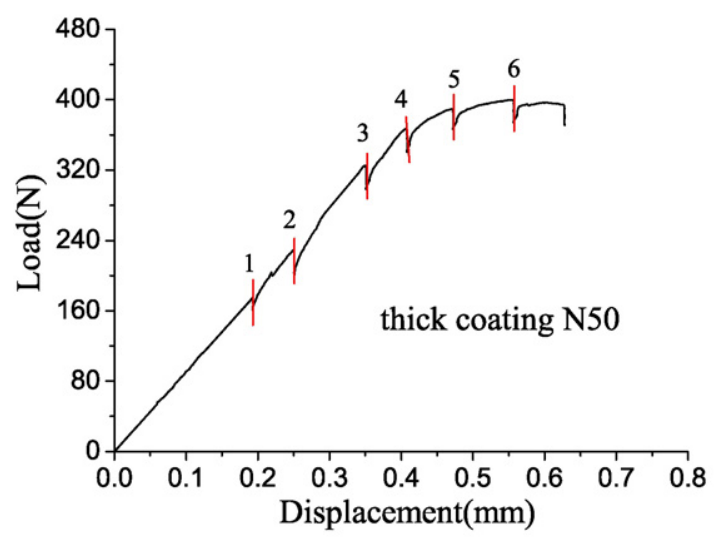

b
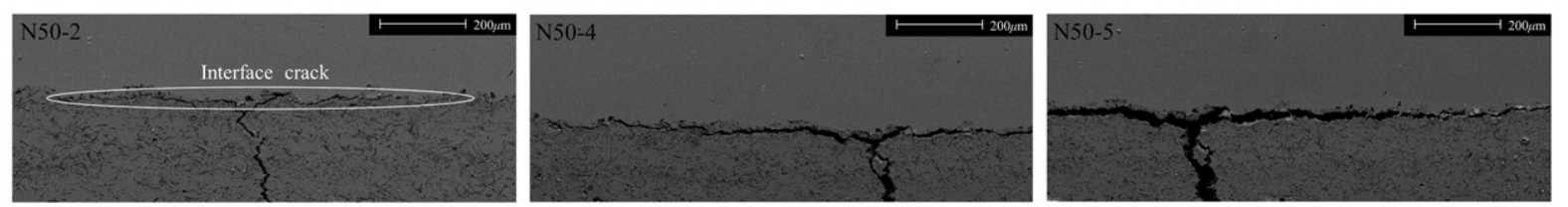

Fig. 3. (a) Load-displacement curves of the nanostructured thick coating sample N50, and (b) the interface crack maps corresponding to the points 2 , 4 , and 5 in the curve.

are the moment of inertia of the substrates and the coatings, respectively, for the neutral axial of the composite beams [11], and $b$ is the width of the specimens, $y$ is the distance from the neutral axial, $h_{c}$ and $h_{s}$ are thickness of the coatings and the substrates, respectively, $S=\frac{E_{s} h_{s}^{2}-E_{c} h_{c}^{2}}{2\left(E_{s} h_{s}+E_{c} h_{c}\right)}$ is the distance between the interface and the neutral axial of the system [11,15]. Note that the definitions of the neutral axial position and the moment of inertia are different from the expressions in Refs. [16-17], but the results of actual equivalent bending stiffness are same. Although only one dimensional stress analysis is carried out, the normalized controlling variable (in fact $P / P_{f}$ ) is a key to check damage evolution with loading. Eq. (1) is effective in the maximum bending moment of the coatings as shown in Fig. 1 for elastic brittle ceramic coatings. Note that the elastic modulus of the coatings is assumed to be a constant as a material property parameter based on the composite beam theory as did in Ref. $[11,15,18]$, the effect of strain on the elastic modulus and the residual deformation are not considered here.

The normalized damage variable $D / D_{f}$ increases with increasing normalized tensile stress $\sigma / \sigma_{f}$ for the thin coatings and shear stress $\tau / \tau_{f}$ for the thick coatings, as shown in Fig. 4(a)-(c) corresponding to the thin coatings (including the nanostructured and conventional thin coatings), the conventional thick coatings, and the nanostructured thick coatings, respectively, the different symbols denote the experimental results of different samples in each figure. It can be seen from the Fig. 4 that the damage initiates at different stress levels with increasing tensile or shear stress, and tends complete as the stress is near the peak value for all kinds of coatings, including the nanostructured, conventional, thin and thick coatings. Considering the fracture mode of instantaneous emergence of the multiple transverse cracks for the thin coatings and the rapid drop of the load for the thick coatings at the interface fracture as discussed above, a catastrophic failure form is naturally associated, after all, the ceramic coating is a brittle material although the plasticity of the substrate covers up the intrinsic characteristics of the coating to some degree. Therefore, a catastrophic failure model is introduced to analyze the damage evolution behavior of the coatings.

(B) Damage model: If a controlling variable of one system, for example, the tensile stress $\sigma$ for the thin coatings (the shear stress for the thick coatings is similar), is continuous and derivative for damage evolution of the system before its catastrophic rupture, it can be expressed as the Taylor expansion of the damage $D$,

$\sigma=\sigma_{f}+\left[\frac{\mathrm{d} \sigma}{\mathrm{d} D}\right]_{D_{f}}\left(D_{f}-D\right)+\frac{1}{2}\left[\frac{\mathrm{d}^{2} \sigma}{\mathrm{d}^{2} D}\right]_{D_{f}}\left(D_{f}-D\right)^{2}+\mathrm{o}\left(D_{f}-D\right)^{2}$

where $\sigma_{f}$ denotes the failure stress corresponding to the fracture of the coatings, the damage variable $D$ is calculated based on the crack length as above defined, and $D_{f}$ represents the complete damage corresponding to $\sigma_{f}$. When $\sigma=\sigma_{f}, D=D_{f}$ based on the damage definition for brittle damage under uni-direction tension, which is consistent with Eq. (3). When the controlling variable is close to the catastrophic point, the damage $D$ increase rapidly and damage rate $R$ tends infinite, i.e., $\lim _{\sigma \rightarrow \sigma_{f}} R=\lim _{\sigma \rightarrow \sigma_{f}} \frac{\mathrm{d} D}{\mathrm{~d} \sigma}=\infty[19-20]$, contrarily, $\lim _{D \rightarrow D_{f}} \frac{\mathrm{d} \sigma}{\mathrm{d} D}=0$, i.e., $\left[\frac{\mathrm{d} \sigma}{\mathrm{d} D}\right]_{D_{f}}=0$, substituting the relation into Eq. (3), and neglecting the high-order term (higher second-order), the damage as a function of the controlling variable can be expressed as

$D=D_{f}-c\left(\sigma_{f}-\sigma\right)^{0.5}$

where $c=\left\{-\frac{1}{2}\left[\frac{\mathrm{d}^{2} \sigma}{\mathrm{d}^{2} D} D_{f}\right\}^{-0.5}\right.$. When $\sigma=\sigma_{0}, D=D_{0}$, taking them into Eq. (4), the damage coefficient $c=\frac{D_{f}-D_{0}}{\sqrt{\sigma_{f}-\sigma_{0}}}$ can be obtained for each sample. Eq. (4) shows that the damage $D$ increases with increasing stress $\sigma$, and the evolution of the damage variable with the controlling variable obeys the power-law relation with the exponent of 0.5 , which is similar to the power-law relation of 0.5 for the residual stress as the function of heat treatment time [21], differently, the residual stress decreases with increasing heating time [21], which is reasonable since the increased damage (crack) releases the strain energy and decreases the residual stress. 
Furthermore, the damage rate (sensitivity) of the ceramic coatings

$R=\frac{\mathrm{d} D}{\mathrm{~d} \sigma}=\frac{c}{2}\left(\sigma_{f}-\sigma\right)^{-0.5}$

can be obtained based on Eq. (4). Eq. (5) shows the damage rate $R$ increases sharply when $\sigma$ is near $\sigma_{f}$, which agrees with the power-law singularity of $R \propto\left(1-U / U_{f}\right)^{-n}$ with the controlling displacement $U$ and the power exponent $n=0.5$ based on the rock catastrophic rupture experiments [20].

\subsection{Comparisons between the experimental results and the damage model}

For the thin coating systems, the normalized damage is expressed as

$\frac{D}{D_{f}}=1-\left[\frac{1-\frac{D_{0}}{D_{f}}}{\sqrt{1-\frac{\sigma_{0}}{\sigma_{f}}}}\right]\left(1-\frac{\sigma}{\sigma_{f}}\right)^{0.5}$

by substituting the damage coefficient $c$ into Eq. (4). For the thick coating systems, the damage is also based on Eq. (6) except substituting the tensile stress by the shear stress. Fig. 4 shows that the experimental measured damage evolution (symbols) is roughly in agreement with the power-law function in terms of Eq. (6) (lines) for all coating samples, including the nanostructured and the conventional thin coatings showed in Fig. 4(a), the conventional thick coatings showed in Fig. 4(b), and the nanostructured thick coatings showed in Fig. 4(c). The parameters used in Eq. (6) for different samples, referring to Tables 1-3 for Fig. 4(a)-(c), respectively, were based on the experimental data. It can be seen from Fig. 4 that the damage is complete when the controlling stress reaches the failure stress. Note that the model is more valid when the damage is near the catastrophic failure point, although the initial damages in different samples occur at different stress levels due to the different microstructure and defects of the coatings. The more valid experimental data are often difficult to be captured for a sample close before the catastrophic failure point, therefore, the experiment data based on different samples were collected to discover the rule.

It can be found from Table 1 that the damage coefficient $c$ (average 1.38 ) of the nanostructured thin coatings $(\mathrm{Nx})$ is larger than $c$ (average 1.01 ) of the conventional thin coatings ( $\mathrm{Mx}$ ), which implies that the damage failure of the nanostructured thin coatings is faster than that of the conventional thin ones. The minimum initial damage is $0.03-$ $0.06\left(D_{0} / D_{f}\right.$ in Tables $\left.1-3\right)$, which is also in agreement with the previous report [9]. Comparing the damage coefficient $c$ of the thin coatings in Table 1 with that of the conventional thick coatings in Table 2, it can be seen that $c$ (average 1.78) of the conventional thick coatings is larger generally and the initial damage is also larger, which means the damage of the conventional thick coating systems is faster, and verifies the experimental phenomenon of the obvious catastrophic characteristics of the load-displacement curves of the thick coating systems. Moreover, the initial damage of the conventional thick coatings occurs at the higher stress level as showed in Fig. 4(b) and Table 2 (average $\tau_{0} / \tau_{f}$ is larger and is closer to 1 ). Comparing the damage coefficient $c$ (average 1.52) of the nanostructured thick coatings in Table 3 with that of the conventional thick coatings in Table 2, it can be seen that $c$ of the conventional thick coatings is also larger mostly, which also means the damage of the conventional thick coatings is faster, which is reasonable since the conventional thick coatings are more similar to the bulk brittle ceramic materials.

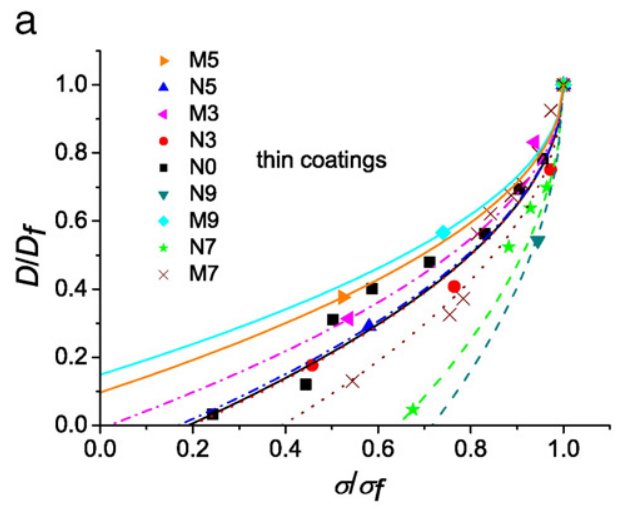

b

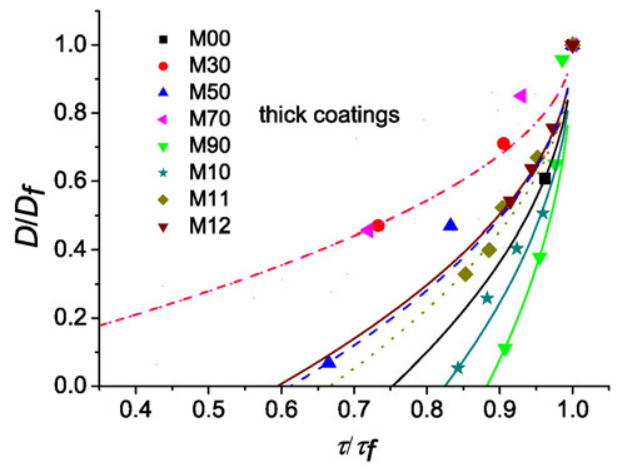

C

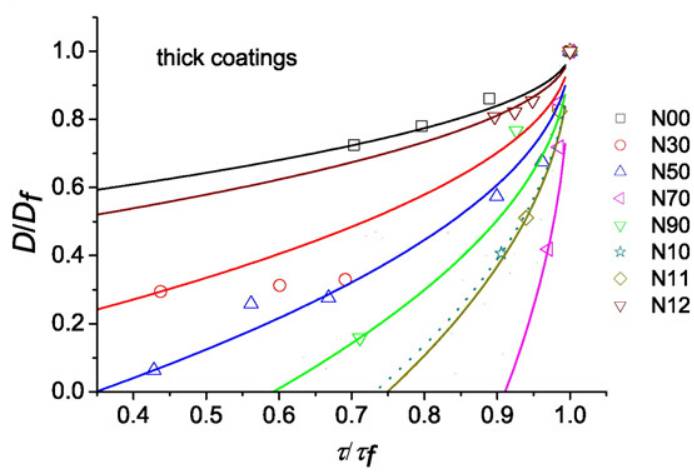

Fig. 4. The normalized damage versus the stress for (a) the thin coatings, (b) the conventional thick coatings, and (c) the nanostructured thick coatings. The symbols are the experimental data, and the lines are based on Eq. (6), where the corresponding parameters for the thin coatings, the conventional and nanostructured thick coatings are in Tables 1, 2, and 3, respectively.

Fig. 5 shows that damage date of the coatings, based on experimental data (symbols) and the following normalized expression for the thin coatings

$R=\left[\frac{1-\frac{D_{0}}{D_{f}}}{2 \sqrt{1-\frac{\sigma_{0}}{\sigma_{f}}}}\right]\left(1-\frac{\sigma}{\sigma_{f}}\right)^{-0.5}$

in terms of Eq. (5). Fig. 5(a)-(c) corresponds to the results of the thin coatings, the conventional and the nanostructured thick coatings, respectively. For the thick coating systems, the tensile stress in Eq. (7) is also replaced by the shear stress. It can be seen from the Fig. 5 that the damage date of the coatings tends infinite as the tensile or shear stress is near the peak value ( $R$ has no meaning at $\sigma^{*}=\sigma / \sigma_{f}=1$ ). Note that Fig. 5 only shows the power-law singularity of the damage sensitivity and can not show the dispersion of the experimental data compared 
Table 1

The parameters in Eqs. (6) and (7) obtained from the experimental measurements of the thin coating samples. $n$ denote the slope of the fitted experimental data by $\ln R=m-n \ln \left(1-\sigma / \sigma_{f}\right)$, i.e., the power exponent of damage rate in Eq. (7), and $m$ corresponds to the intercept.

\begin{tabular}{lrllllllll}
\hline & \multicolumn{1}{l}{ N0 } & N3 & N5 & N7 & N9 & M3 & M5 & M7 & M9 \\
\hline$D_{0} / D_{f}$ & 0.03 & 0.18 & 0.29 & 0.05 & 0.54 & 0.31 & 0.38 & 0.13 & 0.57 \\
$\sigma_{0} / \sigma_{f}$ & 0.24 & 0.46 & 0.58 & 0.68 & 0.94 & 0.54 & 0.52 & 0.54 & 0.74 \\
$c$ & 1.11 & 1.12 & 1.10 & 1.67 & 1.88 & 1.01 & 0.90 & 1.28 & 0.85 \\
$n$ & 0.54 & 0.5 & & 0.5 & & 0.5 & & 0.5 & \\
$m$ & -0.61 & -0.58 & & -0.18 & & -0.31 & & -0.44 & \\
\hline
\end{tabular}

Table 2

The parameters for the conventional thick coating samples.

\begin{tabular}{lcccccccc}
\hline & M00 & M30 & M50 & M70 & M90 & M10 & M11 & M12 \\
\hline$D_{0} / D_{f}$ & 0.61 & 0.47 & 0.07 & 0.46 & 0.11 & 0.05 & 0.33 & 0.54 \\
$\tau_{0} / \tau_{f}$ & 0.96 & 0.73 & 0.67 & 0.72 & 0.91 & 0.84 & 0.85 & 0.92 \\
$c$ & 2.01 & 1.02 & 1.61 & 1.02 & 2.91 & 2.39 & 1.73 & 1.57 \\
$n$ & & 0.5 & 0.5 & 0.5 & 0.5 & 0.5 & 0.5 & 0.5 \\
$m$ & & -0.47 & -0.22 & -0.23 & 0.38 & 0.18 & 0.03 & 0.13 \\
\hline
\end{tabular}

to the model (lines), and the experimental data were obtained based on in Eq. (7) with the parameters resulted from Tables 1-3, for Fig. 5(a)(c) respectively (the symbols are on the corresponding lines). Furthermore, the logarithm relations of the damage rate versus the tensile or shear stress, $\ln R=m-n \ln \left(1-\sigma / \sigma_{f}\right)$, were checked by fitting the experimental data and to obtain the error of the experimental data as shown in Fig. 6 and Tables 1-3, where $m$ and $n$ denote the intercept and the power exponent (slope) of the fitting results, respectively, the blanks in Tables reflect the lack of the corresponding experimental data. $n$ should be 0.5 and $m$ is related to $c$ based on the model (Eq. (7)). The experimental data for each sample were linearly fitted in the logarithm relation, the power exponent $n$ for all validate samples is almost 0.5 as showed in Tables 1-3, but the intercept $m$ of different samples is dispersed.

Fig. 6 shows the logarithm relation of the damage rate versus the tensile or shear stress based on the experimental data (symbols) and the model (lines) resulted from Taylor's expansion with fixed $n(0.5)$ and average $m$ resulted from the fitted experimental results for corresponding samples as shown in Tables 1-3, for Fig. 6(a)-(c) respectively. It can be seen from the figure that the damage rate of the coatings obeys the power-law singularity of -0.5 given by the model. The dispersion for the thin coatings is smaller (see Fig. 6(a)), and the dispersion of the nanostructured thick coatings is larger (see Fig. 6(c)). The damage coefficient $c$ of the nanostructured thick coatings is also more dispersed as shown in Table 3.

\section{Conclusions}

In summary, a damage and catastrophic failure model for ceramic coating systems was developed based on a series of in-situ three-point bending experiments of ceramic coating/alloy substrate samples and the analytic derivation resulted from the Taylor's expansion. The crack evolution was observed and the crack length was used to characterize

Table 3

The parameters for the nanostructured thick coating samples.

\begin{tabular}{lcccccccr}
\hline & N00 & N30 & N50 & N70 & N90 & N10 & N11 & N12 \\
\hline$D_{0} / D_{f}$ & 0.73 & 0.29 & 0.06 & 0.42 & 0.16 & 0.41 & 0.51 & 0.81 \\
$\tau_{0} / \tau_{f}$ & 0.70 & 0.44 & 0.43 & 0.97 & 0.71 & 0.91 & 0.94 & 0.90 \\
$c$ & 0.50 & 0.94 & 1.24 & 3.35 & 1.57 & 1.93 & 2.00 & 0.60 \\
$n$ & 0.5 & 0.5 & 0.5 & 0.5 & 0.5 & & 0.5 & 0.5 \\
$m$ & -1.4 & -0.75 & -0.48 & 0.52 & -0.24 & & -0.005 & -1.2 \\
\hline
\end{tabular}

a

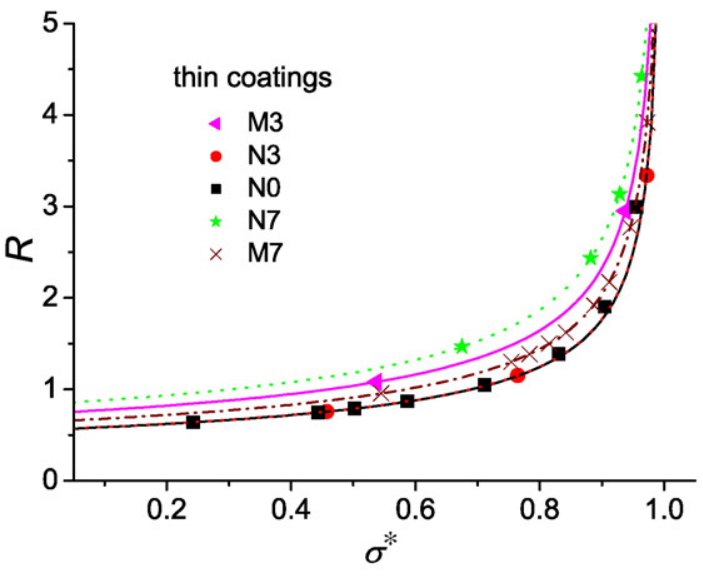

b

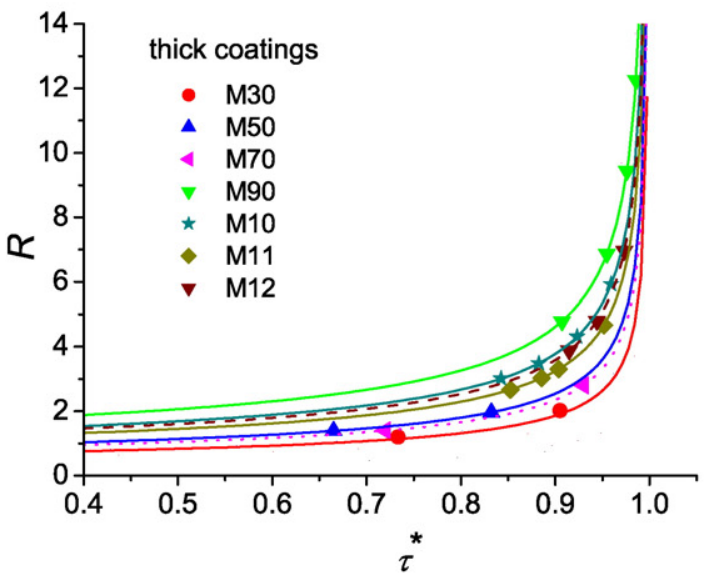

C

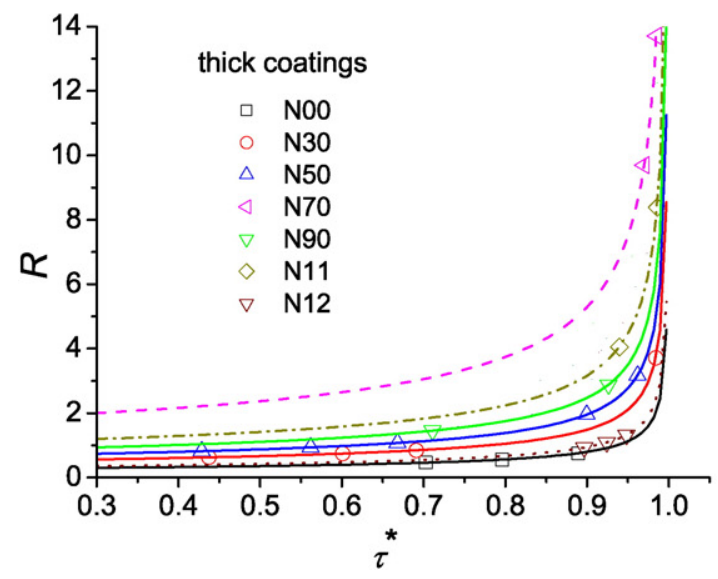

Fig. 5. The damage rate versus the normalized stress for (a) the thin coatings, (b) the conventional thick coatings, and (c) the nanostructured thick coatings with the $\sigma^{*}=\sigma / \sigma_{f}$ and $\tau^{*}=\tau / \tau_{f}$. The symbols are the experimental data, and the lines are based on Eq. (7) with corresponding parameters in Tables 1-3.

the damage, the damage evolution with increasing stress was studied. The results indicate that the damage and failure of the ceramic coatings obey the power-law characteristics with power exponent of 0.5 , which is in agreement with a power law of catastrophic failure of brittle materials. The damage becomes complete and the damage rate tends infinite as the stress is near the peak value. The experimental results for all kinds of studied coatings, including the thin, thick, nanostructured, 
a

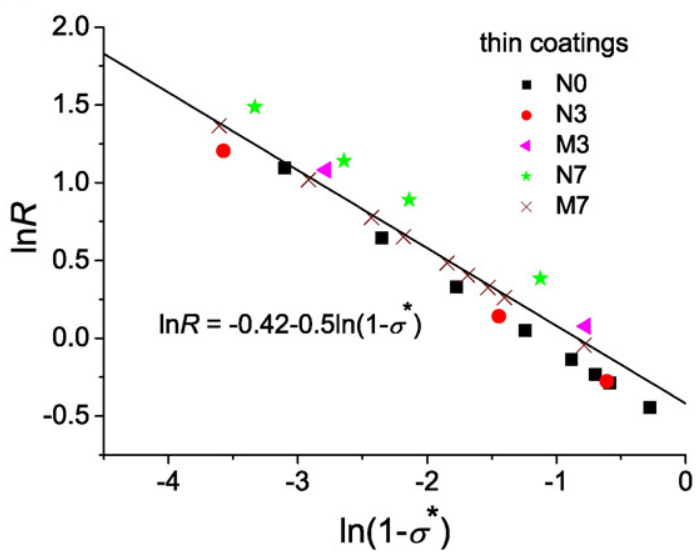

b

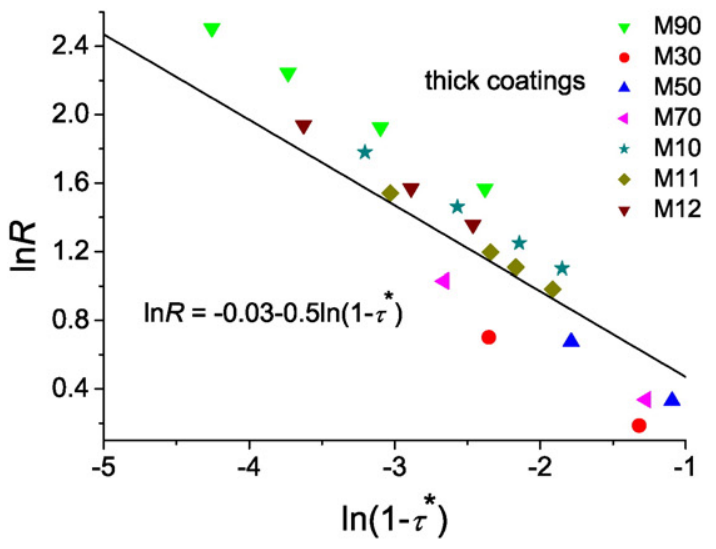

C

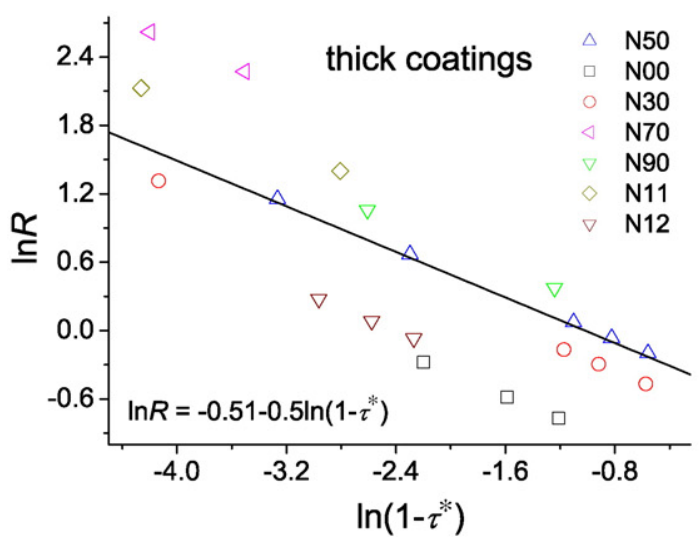

Fig. 6. The logarithm relation of the damage rate versus the stress for (a) the thin coatings, (b) the conventional thick coatings, and (c) the nanostructured thick coatings. The symbols are the experimental data, and the lines are in terms of Eq. (7) with fixed power exponent $n$ of 0.5 and the average intercept $m$ of the fitted experimental data in Tables 1-3 to show deviation of the experimental data.

and conventional coatings, are all consistent with the predictions based on the model.

The study found that the damage of the conventional thick coating systems is faster, so the nanostructurization of the coatings or decreasing thickness of the coatings will improve the performance of the coating systems provided satisfying application. Note that actual failure mechanism of thermal barrier coatings in service may be different from in our experiment. Nevertheless, the model is hopeful to be used to predict the failure of the coating systems if the appropriate controlling variable is selected and the related parameters are known. For example, the model can be extended for studying thermal shock cycling failure characteristics of the coating systems, there the cycling number may be taken as the controlling variable. If the damage coefficient is known, the lifetime can be predicted by the finite damage (crack length) at any cycling and the corresponding cycling number based on the Eq. (4).

\section{Acknowledgements}

This work was supported by the National Basic Research Programs of China (No. 2012CB937500), the NSFC grants of China (Nos. 11372318, 11023001, 11432014, and 91216108), and the CAS/SAFEA International Partnership Program for Creative Research Teams.

\section{References}

[1] N.P. Padture, M. Gell, E.H. Jordan, Thermal barrier coatings for gas-turbine engine applications, Science 296 (2002) 280-284.

[2] Y.C. Zhou, T. Tonomori, A. Yoshida, L. Liu, G. Bignall, T. Hashida, Fracture characteristics of thermal barrier coatings after tensile and bending tests, Surf. Coat. Technol. 157 (2002) 118-127.

[3] L. Qian, S. Zhu, Y. Kagawa, T. Kubo, Tensile damage evolution behavior in plasmasprayed thermal barrier coating system, Surf. Coat Technol. 173 (2003) 178-184.

[4] P. McGuigan, G.A.D. Briggs, V.M. Burlakov, M. Yanaka, Y. Tsukahara, An elasticplastic shear lag model for fracture of layered coatings, Thin Solid Films 424 (2003) 219-223.

[5] M.D. Thouless, Z. Li, N.J. Douville, S. Takayama, Periodic cracking of films supported on compliant substrates, J. Mech. Phys. Solids 59 (2011) 1927-1937.

[6] J. Huang, B.C. Kim, S. Takayama, M.D. Thouless, The control of crack arrays in thin films, J. Mater. Sci. 49 (2014) 255-268.

[7] D. Renusch, M. Schütze, Measuring and modeling the TBC damage kinetics by using acoustic emission analysis, Surf. Coat. Technol. 202 (2007) 740-744.

[8] Trunova, T. Beck, R. Herzog, R.W. Steinbrech, L. Singheiser, Damage mechanisms and lifetime behavior of plasma sprayed thermal barrier coating systems for gas turbines-Part I: Experiments, Surf. Coat. Technol. 202 (2008) 5027-5032.

[9] H. Brodin, E. Robert, S. Johansson, S. Sjostrom, Fracture mechanical modelling of a plasma sprayed TBC system, in: D. Zhu, H.T. Lin (Eds.),Advanced Ceramic Coatings and interfaces IV, Am. Ceramic Soc., OH, USA, 30 2009, pp. 113-124.

[10] L. Yang, Z.C. Zhong, J. You, Q.M. Zhang, Y.C. Zhou, W.Z. Tang, Acoustic emission evaluation of fracture characteristics in thermal barrier coatings under bending, Surf. Coat. Technol. 232 (2013) 710-718.

[11] X.N. Li, L.H. Liang, J.J. Xie, L. Chen, Y.G. Wei, Thickness-dependent fracture characteristics of ceramic coatings bonded on the alloy substrates, Surf. Coat. Technol. 258 (2014) 1039-1047.

[12] M. Zhou, W.B. Yao, X.S. Yang, Z.B. Peng, K.K. Li, C.Y. Dai, W.G. Mao, Y.C. Zhou, C. Lu, In-situ and real-time tests on the damage evolution and fracture of thermal barrier coatings under tension: A coupled acoustic emission and digital image correlation method, Surf. Coat. Technol. 240 (2014) 40-47.

[13] Z. Chen, R.W. Trice, M. Besser, X. Yang, D. Sordelet, Air-plasma spraying colloidal solutions of nanosized ceramic powders, J. Mater. Sci. 39 (2004) 4171-4178.

[14] L.H. Liang, H. Wei, X.N. Li, Y.G. Wei, Size-dependent interface adhesive energy and interface strength of nanostructured systems, Surf. Coat. Technol. 236 (2013) 525-530.

[15] H.X. Deng, H.J. Shi, S. Tsuruoka, Influence of coating thickness and temperature on mechanical properties of steel deposited with Co-based alloy hardfacing coating, Surf. Coat. Technol. 204 (2010) 3927-3934.

[16] J. Malzbender, R.W. Steinbrech, Mechanical properties of coated materials and multi-layered composites determined using bending methods, Surf. Coat. Technol. 176 (2004) 165-172.

[17] T. Wakui, J. Malzbender, R.W. Steinbrech, Strain dependent stiffness of plasma sprayed thermal barrier coatings, Surf. Coat. Technol. 200 (2006) 4995-5002.

[18] A.K. Ray, N. Roy, K.M. Godiwalla, Crack propagation studies and bond coat properties in thermal barrier coatings under bending, Bull. Mater. Sci. 24 (2001) 203-209.

[19] H.Y. Wang, Y.L. Bai, M.F. Xia, F.J. Ke, Microdamage evolution, energy dissipation and their trans-scale effects on macroscopic failure, Mech. Mater. 38 (2006) 57-67.

[20] S.W. Hao, F. Rong, M.F. Lu, H.Y. Wang, M.F. Xia, F.J. Ke, Y.L. Bai, Power-law singularity as a possible catastrophe warning observed in rock experiments, Int. J. Rock Mech. Min. Sci. 60 (2013) 253-262.

[21] B. Heeg, V.K. Tolpygo, D.R. Clarke, Damage Evolution in Thermal Barrier Coatings with Thermal Cycling, J. Am. Ceram. Soc. 94 (2011) S112-S119. 